\title{
Fusion Analysis of Fiber Bragg Grating Sensor and Geotechnical Engineering
}

\author{
Yao Xu, Ping Hu
}

School of Civil Engineering, University of Jinan, Jinan, Shandong, China, 250022

\author{
Keywords: Fiber Grating Sensor, Geotechnical Engineering, Fusion Analysis
}

\begin{abstract}
Optical fiber sensing technology began in the mid-1970s, the earliest applied to the military side. Such as the US Navy with optical fiber sensor technology to monitor the structure of the ship submarine, and research and development of ultra-sensitive fiber Bragg grating sonar system; NASA with optical fiber sensor technology to monitor the performance and structure of aircraft materials and optimization. At the end of the last century, optical fiber sensing technology has been extended to a number of areas, with its unique way of sensing in the defense, aviation, oil, construction, seismic and other aspects have been applied.
\end{abstract}

\section{Introduction}

Compared with electromechanical measurement, fiber optic sensors are easy to realize long-distance signal transmission and measurement control due to intrinsically safe explosion-proof, anti-electromagnetic interference, corrosion resistance, high temperature resistance, small size, light weight, flexible and convenient, especially for harsh environments, Which is widely used. At present, fiber measurement technology in civil engineering, aerospace, ship transport, power industry, petrochemical, medical, nuclear industry and other fields have a very wide range of applications.

In recent years, fiber optic measurement technology in the field of geotechnical engineering has also been more and more widely used. For the bridge, dam, tunnel, high-rise buildings, road pavement and other engineering structure of the safety maintenance, the amount of mechanical monitoring is essential. Fiber optic sensors can be attached to the surface of the engineering structure, can also be buried in the structure of the internal, to monitor its operation process, the formation and development of defects. In addition, a number of Bragg grating-type fiber optic sensors can also be connected in series into a network, the structure of the distributed measurement, which can be obtained within the structure of the displacement field, stress field, strain field, temperature field distribution with time, The sensor signal can be transmitted for a long distance to the central monitoring room for telemetry. International fiber optic sensor research and development originated in the 20th century, 70 years, almost with the development of optical fiber communication technology synchronization. Early "light-intensive" sensor information is measured to measure the intensity of light, so light source ups and downs, fiber bending loss, connection loss and detector aging and other factors will affect the measurement accuracy. Bragg grating sensor (FBG) and Fabry-Pérot cavity sensor (FPI) overcome the main shortcomings of early light intensity sensor, in recent years in the extensive application of geotechnical engineering has achieved very good results. The former is a functional fiber optic sensor, both for point measurement, but also through a transmission fiber to achieve a wide range of line distribution measurement; the latter is a fiber-type optical fiber sensor, generally used for point measurement. This paper only puts on the two kinds of fiber optic sensors as the representative of the technical principle of optical fiber measurement and its application in geotechnical engineering.

\section{Fiber Optic Sensor Working Principle}

Fiber optic transmission through the cable signal, all the optical fiber is made of insulating material, therefore, to avoid electromagnetic, radio and lightning interference, can also be used in high temperature conditions. Another advantage of fiber is the transmission of the signal attenuation is 
very small. In general, the signal loss is about $0.2 \mathrm{~dB} / \mathrm{km}$, which means that more than $90 \%$ of the optical signal can be transmitted to the other end of the cable other than $1 \mathrm{~km}$. For Fabry-Pérot cavity and Bragg grating interferometric sensors, the measured mass is not affected by this attenuation, so a longer fiber cable can be used.

The Fabry-Pérot sensor works on the basis of light interference, which uses a white light source instead of a laser. Many other types of fiber optic sensors use laser light sources, which have a lot of special requirements (such as: warm-up time of the light source, constant temperature, etc.). Sensitivity to temperature and lateral strain is also one of the advantages of the Fabry-Pérot sensor. The Fabry-Pérot strain gauge is now used to illustrate how the Fabry-Pérot sensor works. The basic structure of the sensor is shown in Fig. The Fabry-Pérot sensor is an external sensor with an inductive element at the end of the fiber and internally consists of two opposing half mirrors. The gap between the two mirrors is called the Fabry-Pérot cavity length (Lcavity), when the measured object deformation, the cavity length should also be synchronized changes. The distance between the fixed sensor and the two pads of the capillary is called the sensor measuring the base distance $(\mathrm{Lg})$. The optical signal generated by the white light diode is coupled through the reader into one end of the optical fiber and transmitted to the other end of the fiber connecting the Fabry-Pérot sensor. At this point, a portion of the incident light is reflected by the first half mirror, and the remaining white light passes through the cavity of the Fabry-Pérot sensor and is again reflected back by the second half mirror. Two beams of reflected light interfere with each other, the original incident white light is divided into several different wavelengths of light and return to the reader for signal demodulation.

The conversion of the optical signal into the measured physical quantity is achieved by the Fizeau interferometer and the linear CCD (charge coupled device) set in the reader as shown in Figure 2. The combination of the two is the white light Interactive correlator. The optical signal reflected from the Fabry-Pérot sensor illuminates the Fizeau interferometer along the full width. The Fizeau interferometer consists of a series of spatially distributed interferometers whose thickness changes along the width direction: from nearly zero to tens of microns, that is, from the minimum of the Fabry-Pérot cavity length to the maximum. The light passing through the Fizeau interferometer shows an energy peak along the width of the interferometer, at a location whose thickness is exactly equal to the actual length of the Fabry-Pérot cavity in the sensor.

\section{Application of Geotechnical Engineering}

At present, the application of fiber optic sensors is the most areas of the bridge when the safety monitoring. The Beddington Trail Bridge near Calgary, Canada, was one of the first bridges used in fiber grating sensors (1993). 16 fiber grating sensors were attached to prestressed concrete and carbon fiber composite ribs for long-term monitoring of bridge structures. This was previously considered impossible.

In the summer of 1999, 120 grating sensors were installed on a steel bridge on the Las Cruces 10 Interstate Highway in New Mexico, creating the most used grating sensor on a bridge. The bridge was built in 1970, and now there have been many fatigue cracks. This optical fiber sensor system not only can detect and count the standard vehicle, but also can measure the speed and weight of the vehicle. With this system, it can monitor the structural response, degradation and damage caused by dynamic load, understand the bridge response to traffic Long-term changes. There is a $72 \mathrm{~m}$ span prestressed concrete bridge over the A4 motorway near Dresden, Germany. Meissner et al. Of the University of Dresden buried the Bragg grating into the concrete prism of the bridge to measure the basic linear response under load, And the conventional strain gauges were used to compare the test, which confirmed the feasibility of the application of fiber grating sensors. In the underground engineering and mining engineering, due to excavation and blasting caused by deformation of the surrounding rock, rupture and even instability and damage, therefore, the displacement of the surrounding rock is particularly valued. Germany's GFZ Potsdam has developed a fiber grating sensor for measuring the deformation of the surrounding rock - the FBX measuring anchor, which is embedded in a fiberglass grating in a glass fiber reinforced polymer rock measuring rod, and then 
The measuring bolts are embedded in the surrounding rock mass to monitor the deformation and structural changes of the rock mass in tunnels, chambers, or deep foundations. This sensor is promising for monitoring complex formation information fields, such as displacement, strain, stress, pressure and temperature under harsh environmental conditions. In addition, they have developed a fiber grating seismic imaging system, and in Switzerland, an underground coal mine roadway conducted a field test. The new fiber grating sensor can also be used as a seismic receiver to measure vibration in rock mass. Institute of Dam Safety Monitoring of Changjiang Academy of Sciences has successfully applied the Fabry-Pérot fiber viscometer to the seepage monitoring of the Yangtze River dike and achieved good results. In addition, the theory and method of distributed optical fiber sensor for centralized leak monitoring of Yangtze River dike are also studied. In the underground engineering support, due to the stress relaxation characteristics of the rock itself and the overall displacement of the surrounding rock gradually to the depth of the two reasons, the anchoring force of the anchor after reaching the peak will gradually decrease with time, when the anchor force To a certain level below, the surrounding rock must be reinforced reinforcement. Therefore, the anchor stress must be monitored for long term. At present, the reason why the coal mine roadway often need to be renovated, the reason is that there is no long-term monitoring, timely feedback, timely reinforcement of the roadway, so that even after the roadway damage had to be completely renovated. Due to the various drawbacks of electromechanical measurement, fiber measurement has become a promising long-term monitoring method, its service life of up to 10 a or more, which is the traditional electromechanical measurement can not do. The following is a recent 120-day stress monitoring of the Fabry-Pérot fiber strain sensor and the portable optical fiber reader produced by Roctest, In addition to the many advantages of conventional fiber optic sensors, the Bragg grating-type grating sensor has some advantages that are significantly superior to other fiber optic sensors: (1) this is the most important, that is, its sensing signal is wavelength modulated, (2) to avoid the general interference sensor in the phase measurement is not clear and the inherent reference point of the need; (3) can be used to control the signal is not sensitive to the light source, the optical fiber bending loss, connection loss and the aging of the detector and other factors; Conveniently use wavelength division multiplexing technology in a fiber in series with a number of sensors, to achieve the true sense of the multi-point line distribution measurement. Therefore, once the grating and even optical path in the non-continuous shear deformation or cut, the time domain reflectometer can receive echo, the measurement network can still work in whole or in part, and by time domain analysis and light intensity analysis can be measured The location and size of discontinuous shear deformation in the object are of great significance in the observation of deep landslide such as landslide monitoring and underground engineering. The only drawback is that it is not yet possible to determine the direction of discontinuous deformation.

\section{Conclusion}

Fiber optic sensors can replace the traditional testing techniques in geotechnical engineering in almost all applications. From a technical point of view, it can overcome almost all the shortcomings of traditional geotechnical engineering testing methods. At present, the domestic less use of fiber test technology is mainly due to expensive fiber optic test equipment, only a fiber strain gauge on the need for about 500 yuan, a multi-channel reader needs hundreds of thousands of yuan, which limits the fiber test Application of Technology in Geotechnical Engineering. With the increasing sophistication of domestic technology, the increase in the number of manufacturers, fiber test equipment, the price will be lower and lower, fiber technology to replace the traditional geotechnical engineering testing technology is only a matter of time, which is the trend.

\section{Acknowledgements}

Project: Study on Test and Constitutive Theory of Deformation Characteristics of Silty Sand Considering Rotation of Main Stress Axis

Nature: National Natural Science Foundation of China Youth Project 
Project Number: 51409120

\section{References}

[1] Hu Jun. Application of fiber grating in monitoring prestressed cable force of bridge [J]. Journal of Wuhan University of Technology, 2011

[2] Liu Shengchun, Zhang Dingli, Huang Jun, Zhang Chengping. Study on the Design of Health Monitoring System for Large Shield Tunneling [J]. Editorial Office of Underground Space and Engineering, 2011 (04)

[3] Li Jianhua, Zhang Hong, Liu Youping, Zhou Yefang, Xiong Maodong. Application of optical fiber Bragg grating in settlement monitoring of highway soft foundation [J]. Journal of Central South University, 2011 (02)

[4] Duan Hang. Application of Fiber Bragg Grating Temperature Sensor in Water Temperature Monitoring of Three Gorges Dam [J]. Journal of Yangtze River Scientific Research Institute, 2010 (09)

[5] Xu Xiaodong, Yan Qiao. New Technology for Seepage Monitoring of Peripheral Seams of Shuibuya Concrete Face Rockfill Dam [J]. People's Yangtze River, 2010 (14)

[6] Yang Yanyou, Zhang Feng. Waterway tunnel automatic monitoring system and application [J]. Surveying and Mapping Science, 2010 (S1) 